Paper

\title{
Linear response measurement of single cantilevers in their high amplitude, nonlinear state
}

\author{
Masayuki Sato ${ }^{1 a)}$, Yurina Sada ${ }^{1}$, Shouma Shige ${ }^{1}$, \\ B. Rob Ilic ${ }^{2}$, and A. J. Sievers ${ }^{3}$ \\ ${ }^{1}$ Graduate School of Natural Science and Technology, Kanazawa University \\ Kakumamachi, Kanazawa, Ishikawa 920-1192, Japan \\ ${ }^{2}$ National Institute of Standard and Technology (NIST), Center for Nanoscale \\ Science and Technology \\ 100 Bureau Drive, MS 6201, Gaithersburg, MD 20899-6201, U.S.A. \\ ${ }^{3}$ Laboratory of Atomic and Solid State Physics, Cornell University \\ Ithaca, NY 14853-2501, U.S.A. \\ a) msato153@staff.kanazawa-u.ac.jp
}

Received July 14, 2016; Revised October 30, 2016; Published April 1, 2017

\begin{abstract}
We have used linear response measurements to explore the frequency shift and peak height of the natural frequency resonance of single cantilevers in a nonlinear state versus driver frequency as the bifurcation frequency is approached. Analytical calculations of these properties for a single Duffing resonator are in good agreement in terms of softening of the natural frequency-pump difference frequency and the diverging of its peak amplitude. The deviation of the natural frequency from the analytical curve that is observed for a short cantilever can be ascribed to a large amplitude effect, which, as yet, cannot be explained by simple modifications of the Duffing equation.
\end{abstract}

Key Words: MEMS, cantilever, bifurcation, linear response, natural frequency

\section{Introduction}

Micromechanical systems have attracted much interest because of their useful applications and/or novel nonlinear dynamical behavior $[1,2]$. Studies of a single driven nonlinear oscillator that obeys the Duffing equation have a long history and reveal a variety of phenomena from linear like resonance to chaos [3], depending on the driving conditions. The most well known effect is hysteresis that accompanies a sudden change in amplitude when the driver is swept up or down over a certain frequency range. Because the Duffing resonator is the simplest nonlinear oscillator with these phenomena, this model system has been used for a variety of theoretical studies [4-8], for developing new ideas [9, 10], and for applications [11-13]. The nonlinear dynamics of the single micromechanical cantilever is yet to be measured with precision. 
Starting from the property that the natural frequency (NF) resonance of a nonlinear oscillator corresponds to a homogeneous solution of a driven equation of motion, we apply a low level sinusoidal probe to a single cantilever of different lengths and the vibrational response caused by this perturbation is measured as a spectrum by scanning the probe frequency. Because of the driven-damped condition to maintain steady state, there is one pair of resonance peaks associated with the NF, symmetrically located about the pump frequency and they shift as the bifurcation point is approached.

With two different single cantilever studies we show that the measured NF results of this single resonator agree, in general, with those determined from analytical calculations for a Duffing oscillator. Both sets of experiments show good agreement with the analytically predicted behavior of the NF peak; however, there is a difference in the way the bifurcation frequency is approached for the two different lengths. We ascribed this difference to a larger amplitude effect for the short cantilever, which cannot be explained by a simple modification of the Duffing model equation. This knowledge can be useful in applying the linear probe technique to an array of micro-mechanical oscillator systems [1416]. These studies show that a linear response measurement of a nonlinear system is a very powerful tool with which to investigate with precision the mechanism behind such bifurcation dynamics.

\section{Experimental measurements}

\subsection{Apparatus}

Figure 1 presents a schematic picture of the experimental setup for the linear response measurement. The micro-cantilever sample is set in a vacuum chamber with a piezo-electric driver (PZT). Two oscillators are connected to the PZT, one is the large AC driver (pump) to maintain the large amplitude nonlinear state, and the other is a weak probe with very small AC amplitude for the spectrum measurement. Two different cantilevers were investigated. They are 50 or $55 \mu \mathrm{m}$ long, $15 \mu \mathrm{m}$ width and $300 \mathrm{~nm}$ thick. Each cantilever has a positive nonlinearity, that is, the spring constant increases as the amplitude increases.

To make the linear measurement, first, the pump frequency is increased from a frequency below the linear resonance frequency of the oscillator to one above so that the oscillator reaches a high amplitude state. Then, the probe frequency is scanned across the pump frequency to obtain the required linear response spectrum. The probe signal is analyzed by a lock-in amplifier, which gives sine and cosine response when the cosine probe as used as a reference signal. Typical cosine (real) and sine (imaginary) parts of the response curve are displayed in Fig. 2. The large central peak is due to the pump signal.

\subsection{Long cantilever results}

Figure 3 displays the pump frequency dependence of the probe response. The spectra are ordered from bottom to top with increasing pump frequency. The arrow on the ordinate identifies that particular spectrum when the pump frequency coincides with the linear resonance frequency of the cantilever.

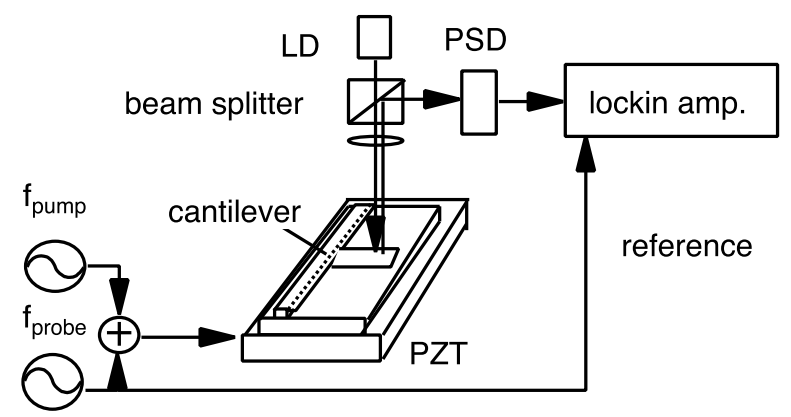

Fig. 1. Experimental setup for the linear response measurement. A cantilever is set in a vacuum chamber and driven vertically by a piezo-transducer (PZT). A laser diode (LD), beam splitter, and position sensitive detector (PSD) are employed the vibrational amplitude measurement. Large amplitude oscillation at $f_{\text {pump }}$ and weak amplitude oscillation at $f_{\text {probe }}$ are induced in the cantilever. The response is analyzed with a lock-in amplifier. 


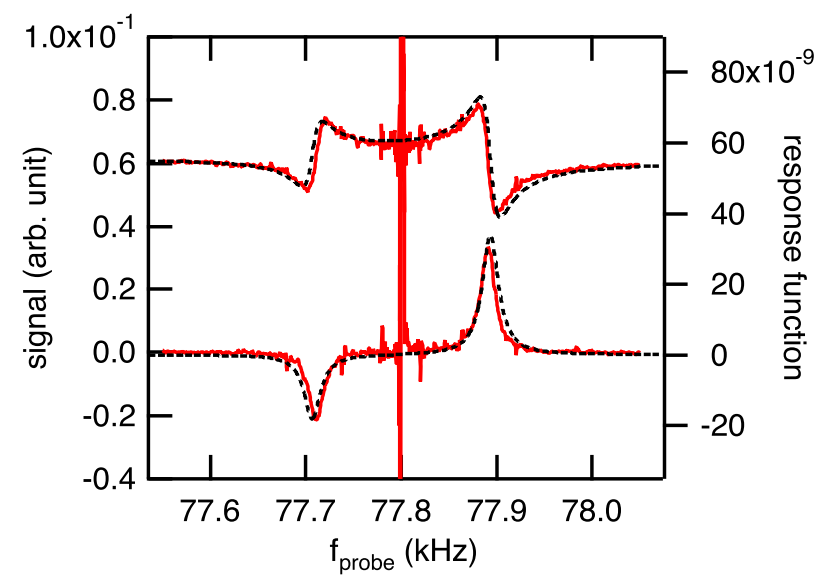

Fig. 2. Cosine (upper) and sine (lower) response spectra for the single cantilever at a high amplitude state. The large peak at the center $(77.8 \mathrm{kHz})$ is due to the large amplitude oscillation by the pump. Relatively wide peaks at both sides are the pair of NF responses. Pump level is $3.9 \mathrm{~V}$, probe level is $21 \mathrm{mV}$. Linear resonance frequency of the $55 \mu \mathrm{m}$ cantilever is $f_{0}=77.519 \mathrm{kHz}$. Dotted curves are response curves calculated by Eq. (2) with parameters $\gamma=1 / \tau=130(1 / \mathrm{s}), \varepsilon=9.87 \times 10^{19}\left(1 / \mathrm{s}^{2} \mathrm{~m}^{2}\right)$ and $\alpha_{1}=5.4 \times 10^{2}\left(\mathrm{~m} / \mathrm{s}^{2}\right)$.

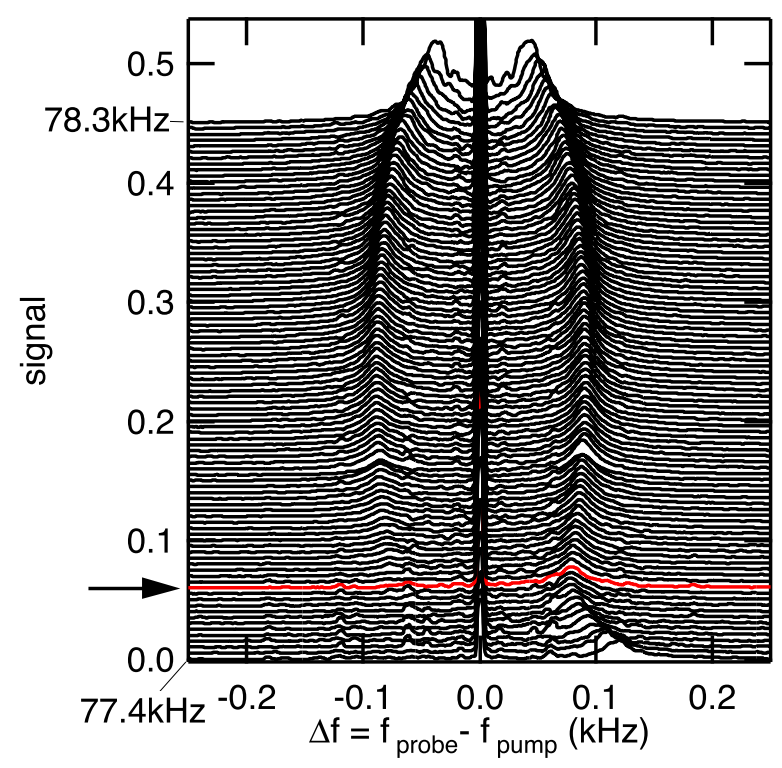

Fig. 3. Response spectra of the long cantilever for various pump frequencies. The PZT amplitude level is $3.9 \mathrm{~V}$, and the probe amplitude is $21 \mathrm{mV}$. Spectra are plotted as a function of difference frequency of the probe and pump, and ordered by pump frequency from bottom $(77.4 \mathrm{kHz})$ to top $(78.3 \mathrm{kHz})$ at $10 \mathrm{~Hz}$ step. The pump frequency spans up to the bifurcation point. As approaching to the upper bifurcation point, the sideband amplitudes diverge and while the sideband frequencies approach the pump. When the pump frequency is $77.4 \mathrm{kHz}$ below the linear resonance frequency $\left(f_{0}=77.519 \mathrm{kHz}\right)$, there is only one peak at $\Delta f=0.12 \mathrm{kHz}(77.52 \mathrm{kHz})$ which is slightly higher than $f_{0}$. When the pump frequency coincides with $f_{0}$ (indicated by the arrow), the peak is shifted at $77.60 \mathrm{kHz}$ which appeared as $\Delta f=0.0815 \mathrm{kHz}$ (red curve).

Below this frequency there is only one resonance peak on the right hand side; but as the pump frequency increases another resonance appears on the left hand side. This peak height increases with the increasing pump frequency and at first the NF gap frequency increases as well. Then at still larger frequencies it decreases as the bifurcation frequency is approached.

The amplitude dependence of the cantilever vs pump frequency, as well as the gap frequency between the NF and the pump and the peak height of the NF are summarized in Fig. 4. The amplitude responses for various pump excitation levels are presented in Fig. 4(a). Figures 4(b) and (c) show 


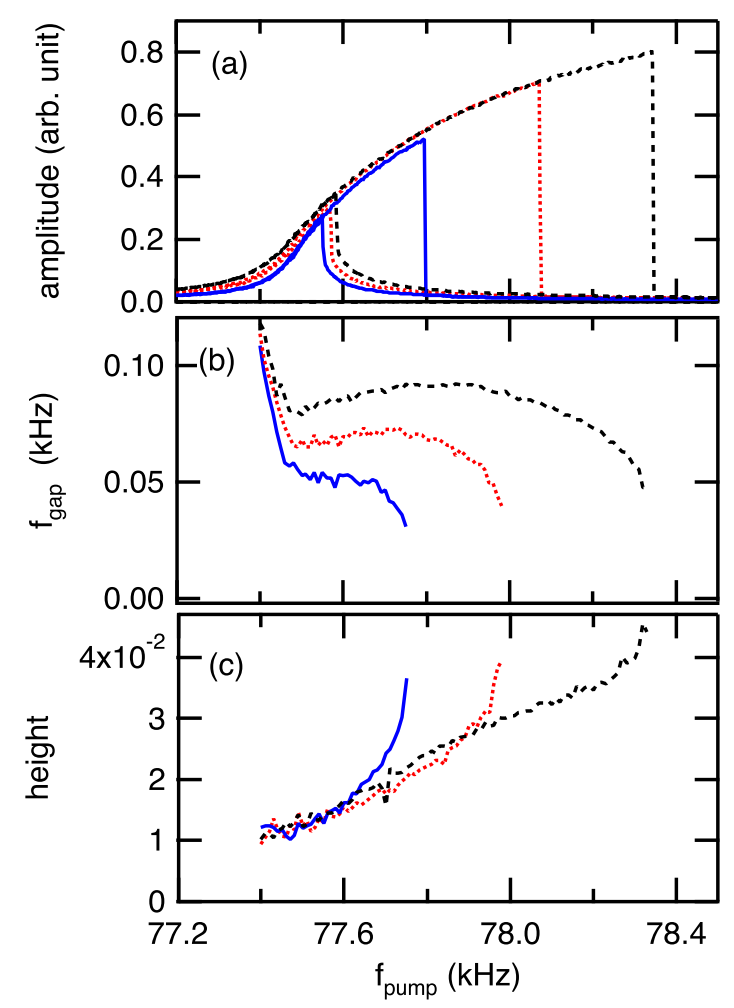

Fig. 4. Summary of the pump response and probe response of the single cantilever. (a) Pump response for various pump amplitudes, 1.8, 2.7, and 3.9V. Solid and dashed curves are for up-scan and down-scan responses, respectively. (b) Gap frequency for different pump levels as a function of the pump frequency.

(c) Peak height of the upper sideband response for different pump levels.

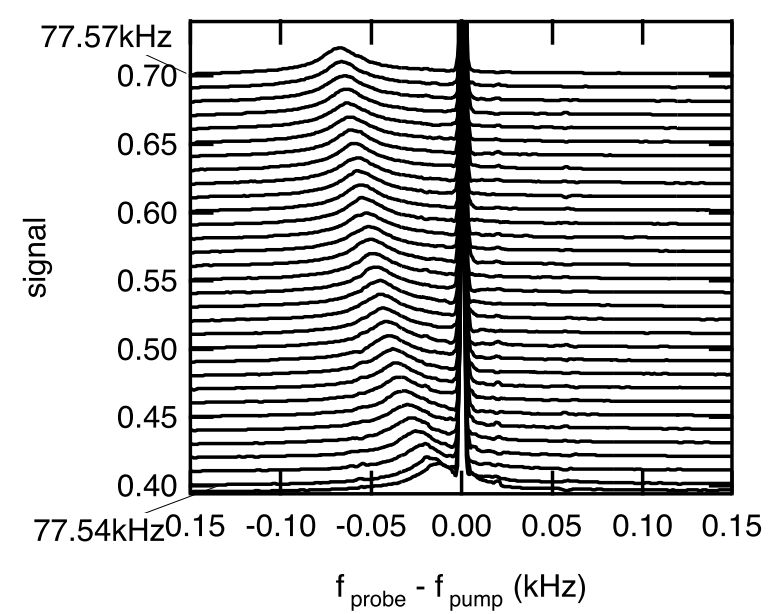

Fig. 5. Probe spectra near the low to high amplitude bifurcation point. Spectra are ordered by the pump frequency from top $(77.570 \mathrm{kHz})$ to the second curve from bottom $(77.540 \mathrm{kHz}$ ) at $1 \mathrm{~Hz}$ step. (The bottom curve is for $77.5395 \mathrm{kHz}$.) The pump frequency was decreased toward the bifurcation frequency $(77.5384 \mathrm{kHz})$. When the pump frequency is far from the linear resonance frequency at $77.519 \mathrm{kHz}$ (top curve), the probe response curve has a peak at the linear resonance frequency. Nearby the bifurcation point, the resonance peak is pulled by the pump frequency. The bifurcation takes place at slightly lower frequency than $77.5395 \mathrm{kHz}$. Pump excitation amplitude is $1.2 \mathrm{~V}$.

the gap frequency and NF peak height dependences, respectively. The gap frequency decreases as the pump frequency approaches the upper bifurcation point, while the peak height tends to diverge. These results are consistent with the analytical results described in Section 3.

Figure 5 shows the linear response spectra for a second bifurcation frequency, which takes place when the pump frequency decreases from above the linear resonance frequency keeping the low amplitude 


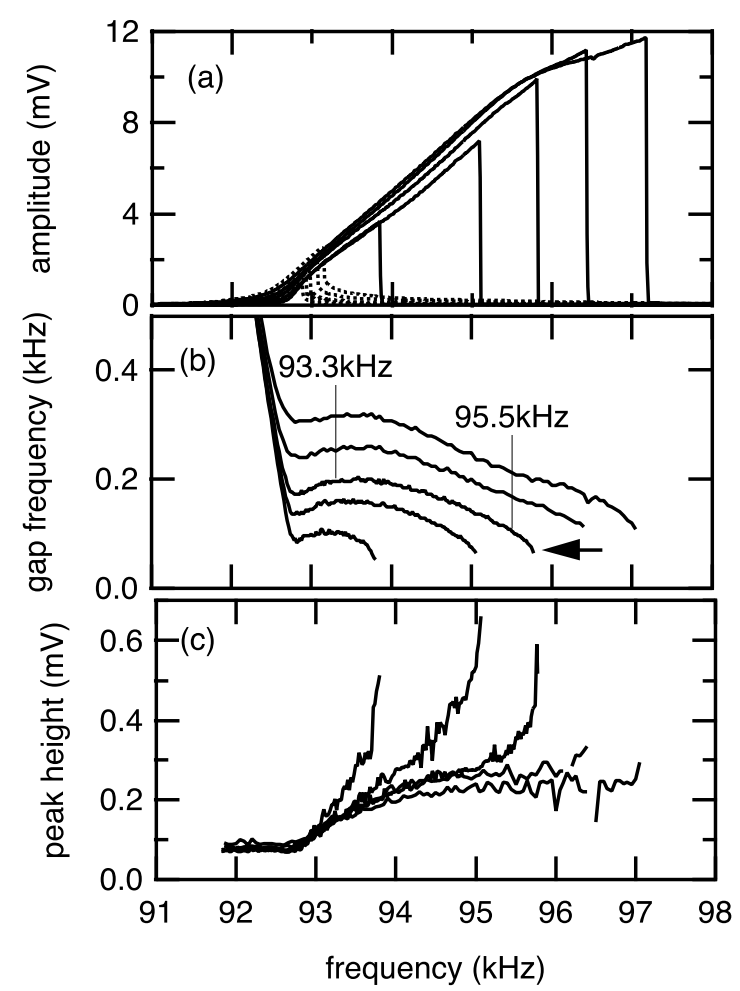

Fig. 6. (a) Short cantilever amplitude vs pump frequency for various excitation levels of the pump. Solid and dashed curves are for up-scan and down-scan respectively. Linear resonance frequency is $92.812 \mathrm{kHz}$. (b) Gap frequency between the NF and the pump vs pump frequency for the same pump excitation levels. (c) Peak height of the sideband response vs pump frequency for the different pump levels.

state. The amplitude suddenly increases at this bifurcation frequency. There is only one NF peak at lower frequencies, because the cantilever is in the low amplitude state, and the pump frequency is higher than the linear resonance frequency. By decreasing the pump frequency to this lower bifurcation point, the cantilever amplitude increases slightly, but still remains in the low amplitude state. Before the transition, the NF peak is pulled by the pump frequency.

\subsection{Short cantilever results}

Slightly different experimental results were observed for the short cantilever. These data are summarized in Fig. 6. The linear resonance frequency is now $92.812 \mathrm{kHz}$. The amplitude response shown in Fig. 6(a) is distorted in the high amplitude state, implying a larger amplitude than Fig. 4(a). This distortion may be an artifact due to saturation of the position sensitive detector. The gap frequency shown in Fig. 6(b) decreases as the bifurcation point is approached; however, the shapes are clearly different from the round shape in Fig. 4(b) at a higher amplitude cases. Figure 6 (c) demonstrates that the divergence of the response peak is observed as well.

\section{Analysis and simulations}

For the model equation of the single Duffing oscillator, we have used

$$
m \ddot{x}+\frac{m}{\tau} \dot{x}+k_{2} x+k_{4} x^{3}=m \alpha_{1} \cos (\Omega t)+m \alpha_{2} \cos (\omega t)
$$

where $m$ is the mass, $\tau$ is the relaxation time, $k_{2}$ and $k_{4}$ are harmonic and quartic spring constants, $\alpha_{1}$ and $\alpha_{2}$ are the driving acceleration amplitude for the pump and probe, and $\Omega=2 \pi f_{\text {pump }}$ and $\omega=2 \pi f_{\text {probe }}$ are the pump and probe angular frequencies, respectively. Values are $m=7.67 \times 10^{-13} \mathrm{~kg}$, $\tau=1 / \gamma=1 / 130(\mathrm{~s}), \sqrt{k_{2} / m}=77519 \times 2 \pi(1 / s)$ and $k_{4}=7.5 \times 10^{7} \mathrm{~N} / \mathrm{m}^{2}$ for $55 \mu \mathrm{m}$ cantilever (the longer one). The mass was estimated from size. The linear spring constant was calculated from the linear resonance frequency, which was measured at a very low excitation level. The nonlinear 
parameter $k_{4}$ was obtained by a fitting started from an initial value estimated by a calculation in Ref. [14].

The analytical complex response function $\tilde{\chi}_{a}$ (function for probe frequency versus probe amplitude curve) was obtained starting from Eq. (2) as follows: [17]

$$
\tilde{\chi}_{a}=\frac{\tilde{\chi}_{0}(\omega)}{1-\frac{9}{16} \varepsilon^{2}|\tilde{A}|^{4} \tilde{\chi}_{0}(\omega) \tilde{\chi}_{0}^{*}\left(\omega^{\prime}\right)}
$$

where $\tilde{\chi}_{0}(\omega)=\frac{1}{\omega_{n l}^{2}-\omega^{2}-i \gamma \omega}, \omega_{n l}^{2}=\omega_{0}^{2}+\frac{3}{2} \varepsilon|\tilde{A}|^{2}, \varepsilon=k_{4} / m, \omega^{\prime}=2 \Omega-\omega, \gamma=1 / \tau$, and $\tilde{A}$ is a complex amplitude caused by the pump driver, $x=\tilde{A} \exp (-i \Omega t) / 2+$ c.c. The peak sideband frequencies can be approximately obtained from the denominator of Eq. (2) by setting its real part equals to zero, ignoring the damping $\gamma$. The result is

$$
\omega=\Omega \pm \frac{1}{\omega_{n l}+\Omega} \sqrt{\left(\omega_{n l}^{2}-\Omega^{2}\right)^{2}-\frac{9}{16} \varepsilon^{2}|\tilde{A}|^{4}} .
$$

The parameters, except the nonlinear parameter $k_{4}$, is estimated from comparison with experiments. Figure 7(a) compares the amplitude response from experiments with analytical calculations. The damping parameter and driving amplitude are obtained from this comparison. Gap frequency and peak heights are well explained by Eqs. (2) and (3) which are plotted in Figs. 7(b) and (c). Figure 8 summarize the pump frequency dynamics of the Duffing resonator for three different pump excitation levels. Curves in Fig. 8(a) represent the analytical pump response solutions. Curves in Fig. 8(b) identify the frequency gap between the NF peak and the pump frequency, as a function of the pump frequency. The linear resonance frequency is $77.519 \mathrm{kHz}$. The gap frequency increases with the pump frequency from the linear resonance frequency, then decreases beyond the middle of the high amplitude frequency region. It approaches zero as the pump frequency reaches the upper bifurcation frequency. The peak height in Fig. 8 (c) increases with increasing pump frequency, and diverging near

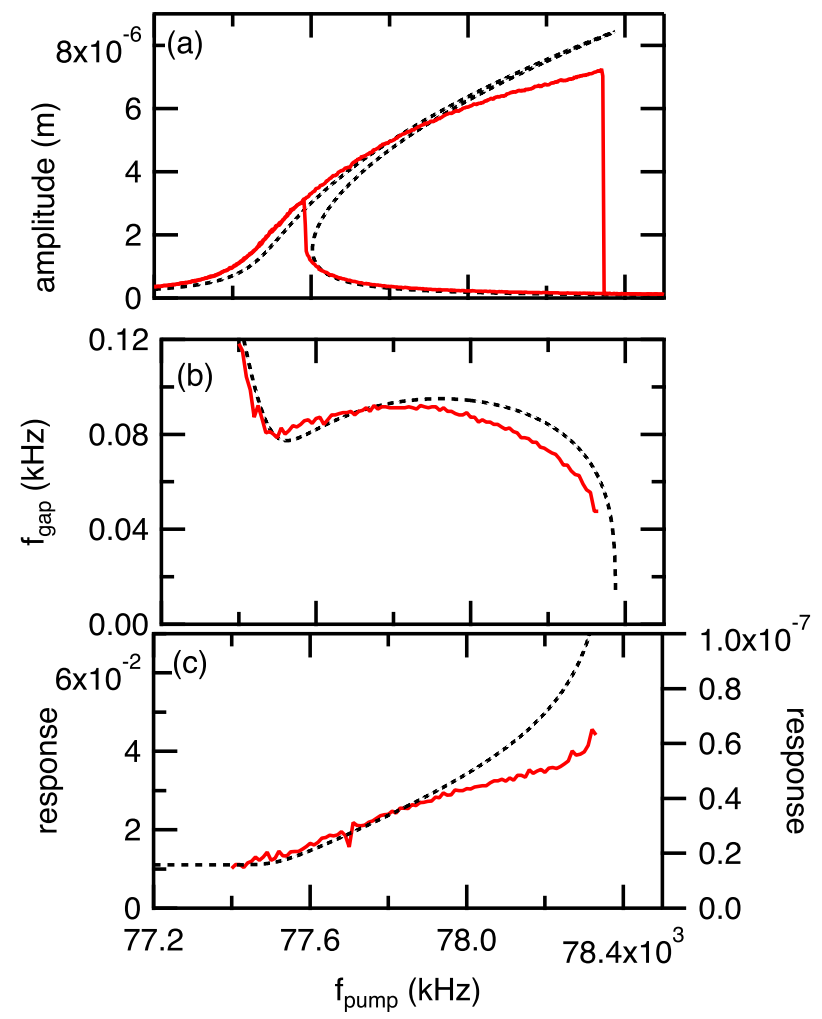

Fig. 7. Comparison of experiments (solid curves) and analytical results (dashed curves). (a) Pump response, (b) gap frequency and (c) peak height for $\alpha_{1}=5.4 \times 10^{2}\left(\mathrm{~m} / \mathrm{s}^{2}\right), \varepsilon=9.87 \times 10^{19}\left(1 / \mathrm{s}^{2} \mathrm{~m}^{2}\right.$ and $\gamma=130(1 / \mathrm{s})$. 

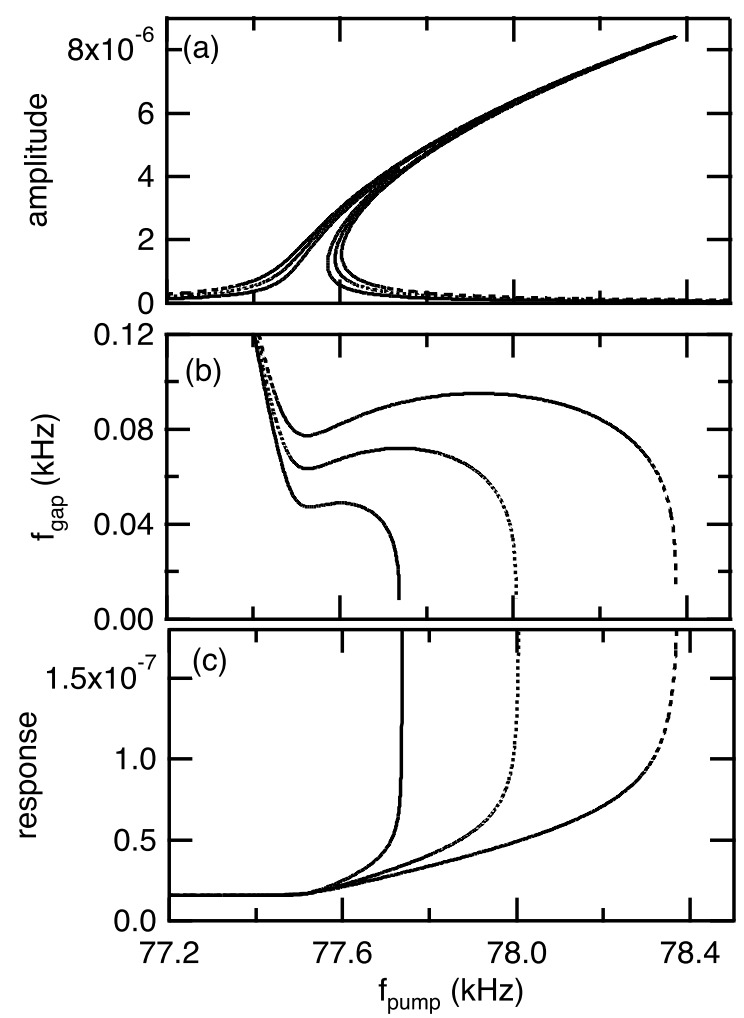

Fig. 8. (a) Nonlinear response of the single Duffing resonator at driving amplitudes $\alpha_{1}=5.4 \times 10^{2}\left(\mathrm{~m} / \mathrm{s}^{2}\right), 4.1 \times 10^{2}\left(\mathrm{~m} / \mathrm{s}^{2}\right)$ and $2.7 \times 10^{2}\left(\mathrm{~m} / \mathrm{s}^{2}\right)$. $\varepsilon=9.87 \times 10^{19}\left(1 / \mathrm{s}^{2} \mathrm{~m}^{2}\right.$ and $\gamma=130(1 / \mathrm{s})$. (b) Analytical sideband gap frequency by Eq. (3). (c) Analytical sideband peak height.

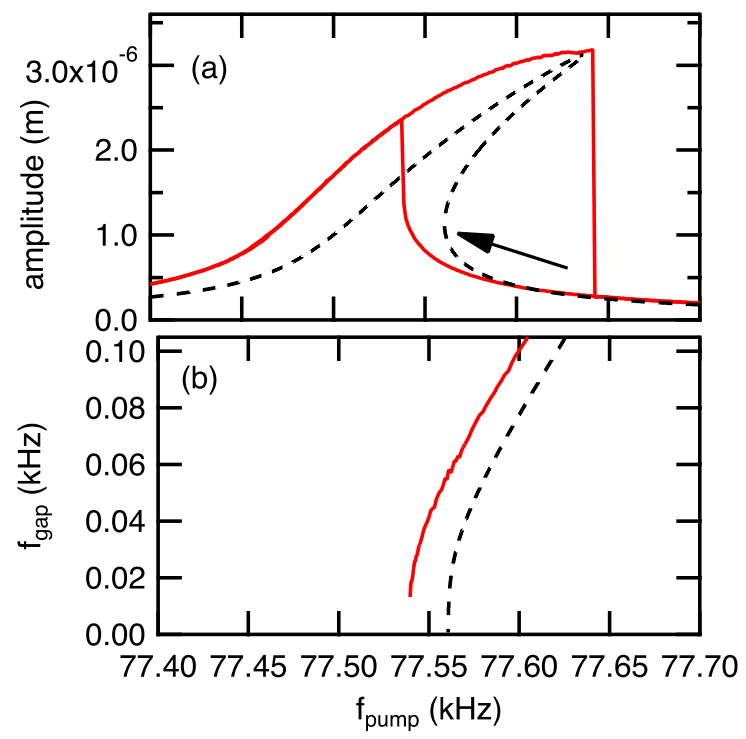

Fig. 9. Summary of the sideband gap frequency for the low-high amplitude bifurcation. (a) Amplitude as a function of the pump frequency. Probe spectra are measured along the curve indicated by the arrow. Dotted curves are analytical results. (b) The gap frequency as a function of the pump frequency. The gap frequency softens suddenly at the bifurcation frequency. Dotted curve is calculated by Eq. (3). Parameters are $\alpha_{1}=2.0 \times 10^{2}\left(\mathrm{~m} / \mathrm{s}^{2}\right), \varepsilon=9.87 \times 10^{19}\left(1 / \mathrm{s}^{2} \mathrm{~m}^{2}\right)$ and $\gamma=130(1 / \mathrm{s})$.

the upper bifurcation frequency.

The comparison between analytical result and experimental results for the low frequency bifurcation is summarized in Fig. 9, which connects the low amplitude state to the high amplitude state. Figure 9(a) shows amplitude as a function of the pump frequency. The pump frequency is decreasing 
as indicated by the arrow. Figure 9(b) shows gap frequency from peaks in Fig. 5 and analytical calculation by Eq. (3).

Similar sideband curves were obtained by Dykman et al. [18], who studied the Duffing oscillator as a model of a stochastic resonance. Since noise plays an important role in the stochastic resonance, they calculated the spectral density of vibration analytically (Eq. (19) in Ref. [18]), and also linear response function (their Eq. (25)). Because of an approximation, their equation is not as simple as Eq. (2) and their peak prediction is distorted by that assumption. Also the gap frequency is related to the stability of the stationary state. For the Duffing equation, stability can be checked by evaluating the perturbed equations in the stationary state $[3,19,20]$.

\section{Discussion}

To compare more precisely the single cantilever results, we evaluate $f_{\text {gap }}^{4}$, because this dependence is known as a predictor of the saddle-node bifurcation for the undamped condition [3]. Three panels as a function of the driver frequency are to be compared in Fig. 10. In this representation, the saddle node bifurcation takes place where the $f_{\text {gap }}$ curve approaches zero at the high frequency location. In all three cases the curves look almost linear near the bifurcation point; however, the experimental curve in (b) for the short cantilever curves up as the pump frequency moves away from that point, while other cases in (a) and (c) curve down. For the experimental short cantilever curve, we plot here the gap curve from Fig. 6(b) (at a middle level excitation indicated by the arrow and the next lower level one). The higher level ones are not used because of the distortion from the round shape.

As shown in Figs. 7 and 9, the analytical predictions and the long cantilever results are in good agreement, for both bifurcations. As shown in Fig. 10 there is a significant difference in the experimental results for the short cantilever. Because the gap frequency and spectra of the long cantilever are well explained by the analytic equations, attention is now focused on the short cantilever experimental condition. First, we compared experimental linewidth, which is proportional to the damping parameter. As shown in Fig. 11, the linear response spectra for these two cantilevers are almost the same, indicating that the damping constant is the same. The fact that the linear resonance frequencies are different should not be a problem. One difficulty of the experimental setup shown in Fig. 1 is that the
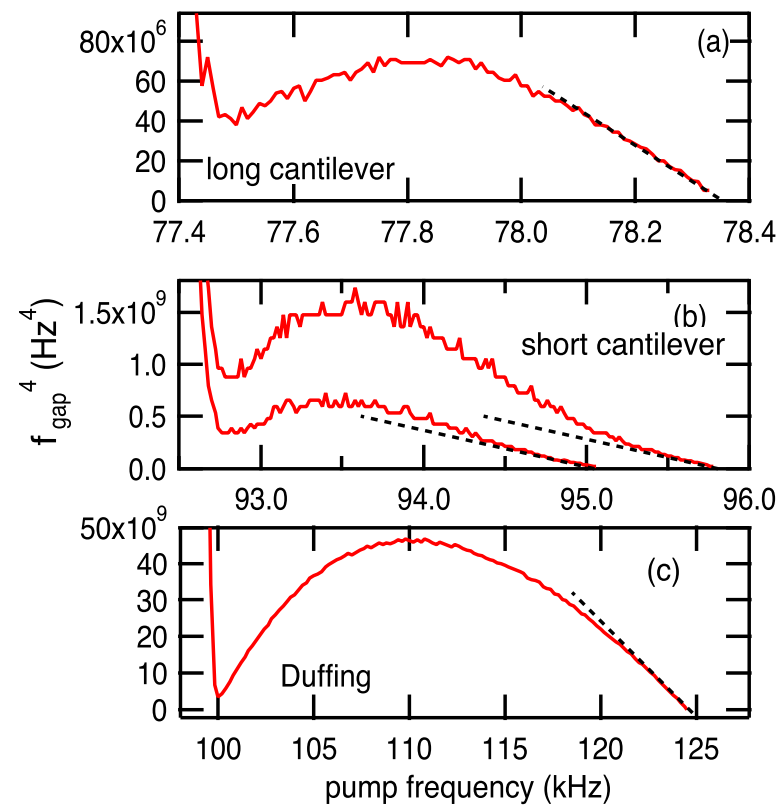

Fig. 10. Predictor of the saddle-node bifurcation, $f_{\text {gap }}^{4}$, vs pump frequency for four cases. (a) Experimentally measured single long cantilever, (b) Experimentally measured single short cantilever, (c) Analytically calculated single cantilever. Two curves in panel (b) are from the middle level excitation data in Fig. 6(b). The approach to zero on the high frequency side is the signature of the saddle-node bifurcation. Dashed lines are guide to eye. 


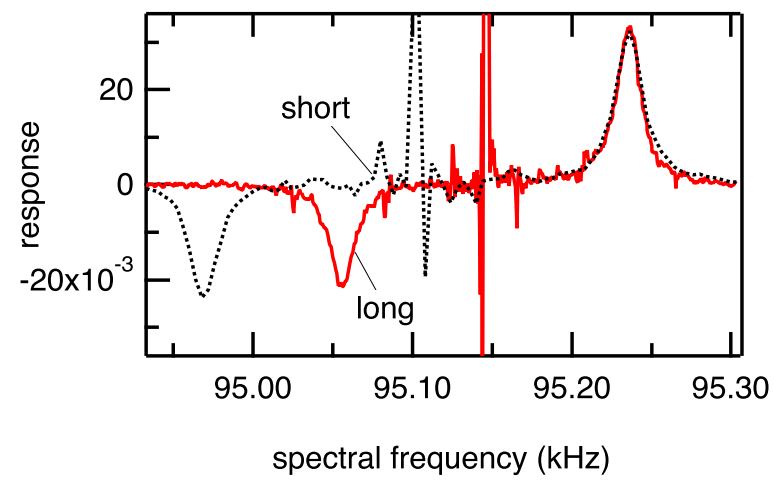

Fig. 11. Linear response spectra for short (dashed) and long (solid) cantilever samples. Curve for the long cantilever was shifted to overlap the two NF peaks. Damping parameter $\gamma$ of these cantilevers are the same because the line width of the sideband responses are the same.

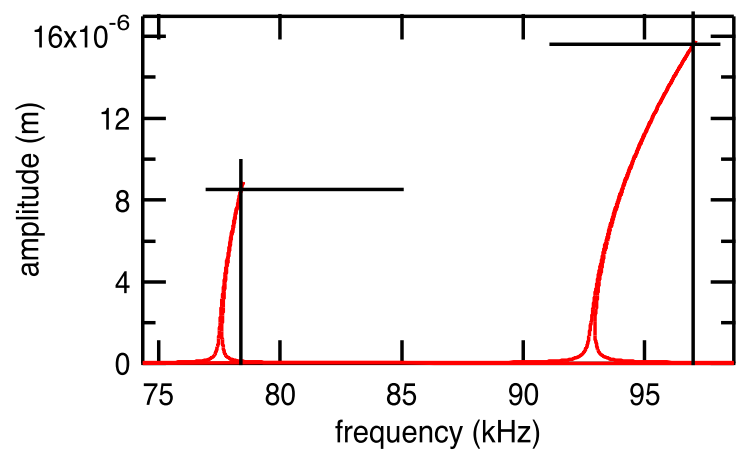

Fig. 12. Amplitude differences between two cantilevers, estimated from the nonlinear parameter and frequency shifts. Vertical lines are the maximum pump frequencies for each cases, and horizontal lines are corresponding amplitudes. The long cantilever was less excited compared with the short cantilever. The amplitude of shorter cantilever was two times larger than the long cantilever.

amplitude cannot be measured accurately. We estimated the amplitude from the difference frequency between the linear resonance and the pump. It requires knowledge of the nonlinear parameter $k_{4}$. We used equations in the Ref. [14] appendix to estimate them.

Figure 12 shows amplitude curve for these two cases indicating that the maximum amplitude for the short cantilever in Fig. 6 may be twice larger than for the long cantilever in Fig. 4. For the middle level data in Fig. 6(b), the amplitude may be still 1.5 times larger than for the long cantilever case. This large amplitude variation may cause the difference in the results. Supporting this proposal, the same plot for one level lower amplitude case in Fig. 6(b) shows smaller up-turn than the curve in Fig. 10(b).

Because the analytical method based on the Duffing equation has no amplitude limit, we conclude that there is a physical difference between the real cantilever systems and our model equations of motions, and this difference becomes conspicuous at larger amplitudes. A likely candidate is nonlinear damping [21], with larger damping for the larger amplitude state. To test this idea, we compared the linewidth for the short cantilever case indicated by two vertical lines in Fig. 6(b). As shown in Fig. 13, the line-width didn't change in the large amplitude state. We have also tested the nonlinear damping by simulations; however, the only cases that showed similar upturns were with negative nonlinear damping, that is, smaller damping at a larger amplitude. Because of no change of line-width in Fig. 13, and no physics supporting negative damping, we conclude that the nonlinear damping is not the source of the up-turn.

We have tested several models, such as adding a cubic potential term $k_{3} x^{2}$, adding 6 th power potential term $k_{6} x^{5}$, modifying the linear spring by temperature effect $k_{2} \rightarrow k_{2} \pm \delta T$ or $k_{2} \rightarrow k_{2} \pm(\delta T)^{2}$ which is simulated by 


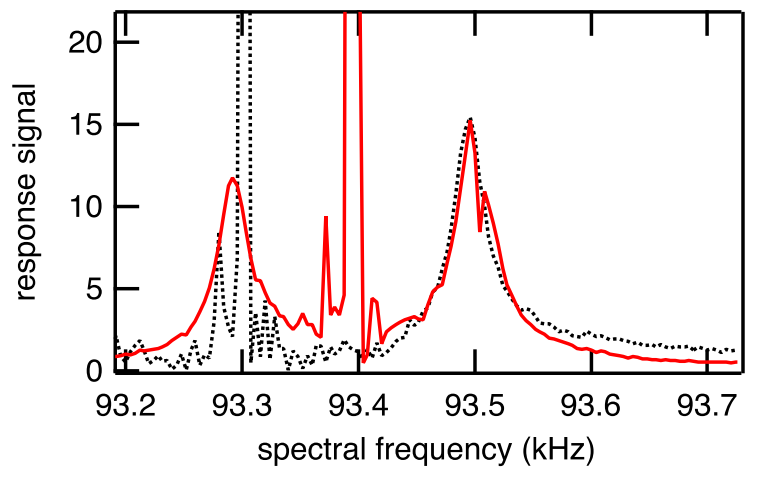

Fig. 13. Response spectra at 93.3 (dashed) and $95.5 \mathrm{kHz}$ (solid) of the short cantilever. These frequencies are indicated by vertical lines in Fig. 6(b). Note that the line width at $95.5 \mathrm{kHZ}$ is the same at $93.3 \mathrm{kHz}$. Curve for $95.5 \mathrm{kHz}$ was shifted for comparison.

$$
\frac{d T}{d t}=-\frac{1}{5 \tau}+x^{2}
$$

We found that none of these possibilities reproduces the upturn in Fig. 10(b). The cantilever equation given by Eq. (1) is derived from Euler-Bernoulli continuous partial differential equation assuming a cantilever shape function obtained at a linear limit. Such assumption is not realistic for the large amplitude case. In fact a large amplitude for the shorter cantilever means a large bending of the shape.

\section{Summary}

For the single cantilever, positive nonlinear case studied here, the saddle node bifurcation takes place at the high frequency side of the amplitude spectrum where it drops suddenly as demonstrated in Figs. 4(a) and 6(a). We have found that the measured frequency shift and peak height of the NF resonance for the long single cantilever is in good agreement with analytical calculations, as the bifurcation frequency is approached, i.e., the gap frequency decreases and the peak height diverges. Experiments for the short cantilever show a difference with the analytic case in that the gap frequency deviates from the analytical curve at a large amplitude, indicating that larger bending may cause the deviation from the Duffing model.

\section{Acknowledgments}

M. S. was supported by JSPS-Grant-in-Aid for Scientific Research No. 25400394. A. J. S. was supported by Grant NSF-DMR-0906491.

\section{References}

[1] E. Kenig, R. Lifshitz, and M.C. Cross, Phys. Rev. E, vol. 79, p. 026203, 2009.

[2] J.F. Rhoads, S.W. Shaw, and K.L. Turner, Journal of Dynamic Systems, Measurement and Control, Transactions of the ASME, vol. 132, p. 1, 2010.

[3] J.M.T. Thompson and H.B. Stewart, Nonlinear Dynamics and Chaos. John Wiley \& Sons, Chichester, 1987.

[4] I. Serban, M.I. Dykman, and F.K. Wilhelm, Phys. Rev. A, vol. 81, p. 022305, 2010.

[5] A. Chatterjee, Int. J. Mech. Sci., vol. 52, p. 1716, 2010.

[6] A. Elias-Zuniga, Nonlin. Dyn., vol. 45, p. 227, 2006.

[7] B. Yurke, D.S. Greywall, A.N. Pargellis, and P.A. Busch, Phys. Rev. A, vol. 51, p. 4211, 1995.

[8] A.G. Shagalov, J.J. Ramsmussen, and V.Naulin, J. Phys. A: Math. Theor., vol. 42, p. 045502, 2009.

[9] J. Fajans and L. Friedland, Am. J. Phys., vol. 69, p. 1096, 2001.

[10] K.M. Murch, R. Vijay, I. Barth, J.A. Aumentado, L. Friedland, and I. Siddiqi, Nat. Phys., vol. 7, p. 105, 2011. 
[11] R.B. Karabalin, R. Lifshitz, M.C. Cross, M.H. Matheny, S.C. Masmanidis, and M.L. Roukes, Phys. Rev. Lett., vol. 106, p. 094102, 2011.

[12] I. Kozinsky, H.W.C. Postma, O. Kogan, A. Husain, and M.L. Roukes, Phys. Rev. Lett., vol. 99, p. 207201, 2007.

[13] J.S. Aldridge and A.N. Cleland, Phys. Rev. Lett., vol. 94, p. 156403, 2005.

[14] M. Sato, B.E. Hubbard, and A.J. Sievers, Rev. Mod. Phys., vol. 78, p. 137, 2006.

[15] M.K. Zalalutdinov, J.W. Baldwin, M.H. Marcus, R.B. Reichenbach, J.M. Parpia, and B.H. Houston, Appl. Phys. Lett., vol. 88, p. 143504, 2006.

[16] S. Krylov, S. Lulinsky, B.R. Ilic, and I. Schneider, Appl. Phys. Lett., vol. 105, p. 071909, 2014.

[17] M. Sato, S. Imai, N. Fujita, W. Shi, Y. Takao, Y. Sada, B.E. Hubbard, B. Ilic, and A.J. Sievers, Phys. Rev. E, vol. 87, p. 012920, 2013.

[18] M.I. Dykman, D.G. Luchinsky, R. Mannella, P.V.E. McClintock, N.D. Stein, and N.G. Stocks, Phys. Rev. E, vol. 49, p. 1198, 1994.

[19] A.H. Nayfeh and D.T. Mook, Nonlinear Oscillations. John Wiley \& Sons, New Yor, 1979.

[20] D.W. Jordan and P. Smith, Nonlinear Ordinary Differential Equations. Oxford University Press, Oxford, NY, 2007.

[21] S. Zaitsev, O. Shtempluck, E. Buks, and O. Gottlieb, Nonlinear Dynamics, vol. 67, p. 859, 2012. 